\title{
Assessment of gait symmetry improvements in national athletes after anterior cruciate ligament reconstruction during rehabilitation
}

\begin{abstract}
This study aimed to quantify changes in gait parameters and their symmetries among athletes with anterior cruciate ligament (ACL) reconstructions during a rehabilitation program. Twenty-two national players with ACL reconstructions and 15 healthy athletes were recruited. The gait data were collected between postoperative weeks 4-5, 8-9 and 12-13 using a three-dimensional motion analysis system. The spatio-temporal gait parameters and symmetry indexes (SIs) were evaluated for the patients and the control group. One-way and repeated-measures multivariate analysis of variance were used to analyse the data. The results demonstrated significant differences among spatio-temporal $(\mathrm{P}<0.001)$ and SIs $(\mathrm{P}=0.007)$ of patients for Test 1 and the control group. Repeated measure analysis revealed significant changes in the linear combinations of spatio-temporal gait variables $(\mathrm{P}=0.002)$ and SIs $(\mathrm{P}=0.043)$ over time. The injured limb's step length, cadence and weight acceptance time presented significant improvement across time $(\mathrm{P}<0.001)$. Moreover, the SI of the stance time was reduced significantly by $46.48 \%$ ( $\mathrm{P}=0.004)$ among SI parameters. After three months, no significant differences were found between patients and healthy controls for the measured gait components $(\mathrm{P}>0.05)$. The rehabilitation program allowed national athletes to restore symmetry in spatio-temporal gait parameters toward the control group's range 12-13 weeks post-reconstruction.
\end{abstract}

Keyword: Gait analysis; Symmetry; Anterior cruciate ligament; Reconstruction; Spatiotemporal 